\title{
Spectroscopic Study of Globular Clusters in the Halo of M31 with Xinglong 2.16m Telescope II: Dynamics, Metallicity and Age *
}

\author{
Zhou Fan ${ }^{1}$, Ya-Fang Huang ${ }^{1,2}$, Jin-Zeng $\mathrm{Li}^{1}, \mathrm{Xu}_{\text {Zhou }}{ }^{1}$, Jun $\mathrm{Ma}^{1}$ and Yong-Heng Zhao ${ }^{1}$ \\ 1 Key Laboratory of Optical Astronomy, National Astronomical Observatories, Chinese Academy of \\ Sciences, Beijing 100012, China; zfan@bao.ac.cn \\ 2 Graduate University of Chinese Academy of Sciences, Beijing 100049, China
}

Received [year] [month] [day]; accepted [year] [month] [day]

\begin{abstract}
In our Paper I, we performed the spectroscopic observations of 11 confirmed GCs in M31 with the Xinglong 2.16m telescope and we mainly focus on the fits method and the metallicity gradient for the M31 GC sample. In this paper, we analyzed and discussed more about the dynamics, metallicity and age, and their distributions as well as the relationships between these parameters. In our work, eight more confirmed GCs in the halo of M31 were observed, most of which lack the spectroscopic information before. These star clusters are located far from the galactic center at a projected radius of $\sim 14$ to $\sim 117 \mathrm{kpc}$, which are more spatially extended than that in the previous work. The Lick absorption-line indices and the radial velocities have been measured primarily. Then the ages, metallicities $[\mathrm{Fe} / \mathrm{H}]$ and $[\alpha / \mathrm{Fe}]$ have been fitted by comparing the observed spectral feature indices and the SSP model of Thomas et al. in the Cassisi and Padova stellar evolutionary tracks, respectively. Our results show that most of the star clusters of our sample are older than $10 \mathrm{Gyr}$ except B290 $5.5 \mathrm{Gyr}$, and most of them are metal-poor with the metallicity $[\mathrm{Fe} / \mathrm{H}]<-1$, suggesting that these clusters were born at the early stage of the galaxy's formation. We find that the metallicity gradient for the outer halo clusters

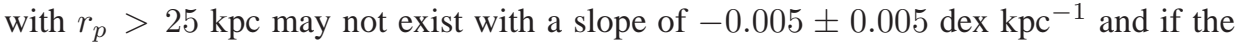
outliers G001 and H11 are excluded, the slope dose not change significantly with a value of $-0.002 \pm 0.003 \mathrm{dex} \mathrm{kpc}^{-1}$. We also find that the metallicity is not a function of age for the GCs with age $<7$ Gyr while for the old GCs with age $>7$ Gyr there seems to be a trend that the older ones have lower metallicity. Besides, We plot metallicity distributions with the largest sample of M31 GCs so far and it shows the bimodality is not significant and the number of the metal-poor and metal-rich groups becomes comparable. The spatial distributions shows that the metal-rich group is more centrally concentrated while the metal-poor group is occupy a more extended halo and the young population is centrally concentrated while the old populaiton is more extended spatially to the outer halo.
\end{abstract}

Key words: galaxies: individual (M31) - galaxies: star clusters — globular clusters: general — star clusters: general

\section{INTRODUCTION}

One way to better understand the formation and evolution of the galaxies is through detailed studies of globular clusters (GCs), which are often considered to be the fossils of galactic formation and evolution

\footnotetext{
* Supported by the National Natural Science Foundation of China.
} 
processes, since they formed at the early stages of their host galaxies' life cycles (Barmby et al., 2000). GCs are densely packed, very luminous, which usually contains several thousands to approximately one million stars. Therefore, they can be detected from great distances and are suitable as probes for studying the properties of extragalactic systems. Since the halo globular clusters (HGCs) are located far away from the galaxy center, they are very important and useful to study the dark matter distribution of the galaxy. Besides, since the HGCs are far from the galaxy center, the background of galaxy becomes much lower, which makes the observations much easier, compared to the disc GCs in the projected direction of galaxies.

As the nearest ( $\sim 780 \mathrm{kpc})$ and large spiral galaxy in our Local Group, M31 (Andromeda) contains a great number of GCs from $460 \pm 70$ (Barmby \& Huchra, 2001) to 530 (Perina et al., 2010), which is an ideal laboratory for us to study the nature of the HGCs. A great many of new M31 HGCs have been discovered in the recent years, which are important to study the formation history of M31 and its dark matter content. Huxor et al. (2004) discovered nine previously unknown HGCs of M31 using the INT survey. Subsequently, Huxor et al. (2005) found three new, extended GCs in the halo of M31, which have characteristics between typical GCs and dwarf galaxies. Mackey et al. (2006) reported four extended, low-surface-brightness clusters in the halo of M31 based on Hubble Space Telescope/Advanced Camera for Surveys (ACS) imaging. These star clusters are structurally very different from typical M31 GCs. On the other side, since they are old and metal-poor, they look like the typical Milky Way GCs. Huxor (2007) found 40 new extended GCs in the halo of M31 (out to $\sim 100 \mathrm{kpc}$ from the galactic center) based on INT and CFHT imaging. These extended star clusters in the M31 halo are very similar to the diffuse star clusters (DSCs) associated with early-type galaxies in the Virgo Cluster reported by Peng et al. (2006) based on the ACS Virgo Cluster survey. Indeed, the evidence shows that DSCs are usually fainter than typical GCs. Later, Mackey et al. (2007) reported 10 outer-halo GCs in M31, at $\sim 15 \mathrm{kpc}$ to $100 \mathrm{kpc}$ from the galactic center, eight of which were newly discovered based on deep ACS imaging. The HGCs in their sample are very luminous, compact with low metallicity, which are quite different from their counterparts in our Galaxy. More recently, Ma et al. (2010) constrained the age, metallicity, reddening and distance modulus of B379, which also is an HGCs of M31, based on the multicolor photometry.

In Fan et al. (2011) (hereafter Paper I) we observed 11 confirmed star clusters, most of which are located in the halo of M31, with the OMR spectrograph on 2.16m telescope at Xinglong site of National Astronomical Observatories, Chinese Academy of Sciences, in fall of 2010. We estimated the ages, metallicities, $\alpha$-elements with the SSP models as well as the the radial velocities and they found that most of the halo clusters are old and metal-poor, which were supposed to be born at the early stage of the galaxy formation history. In this paper, we will continue the study of the HGCs of M31 with the same instruments and a larger sample. This allows us to be able to better understand the properties of the M31 outer halo. This paper is organized as follows. In 92 we describe how we selected our sample of M31 GCs and their spatial distribution. In $\$ 3$, we reported the spectroscopic observations with 2.16 $\mathrm{m}$ telescope and how the data was reduced and the radial velocities and Lick indices were measured and calibrated. Subsequently, in $\$ 4$ we derive the ages, metallicities and $\alpha$-element with $\chi^{2}-$ minimization fitting. We also discuss our final results on the metallicity distribution in the M31 halo. Finally, we summarized our work and give our conclusions in $\$ 5$

\section{SAMPLE SELECTION}

The sources were selected from the updated Revised Bologna Catalogue of M31 globular clusters and candidates (RBC v.4, available from http://www.bo.astro.it/M31; Galleti et al., 2004, 2006, 2007, 2009), which is the latest and most comprehensive M31 GC catalogue so far. The catalogue contains 2045 objects, including 663 confirmed star clusters, 604 cluster candidates, and 778 other objects that were previously thought to be GCs but later proved to be stars, asterisms, galaxies, or HiI regions. Indeed, many of the halo clusters were from Mackey et al. (2007), who reported $10 \mathrm{GCs}$ in the outer halo of M31 from their deep ACS images, of which eight were detected for the first time (see for details in 11 ). In our work, our sample clusters are completely selected from RBC v.4. We selected the confirmed and 
luminous clusters as well as being located as far as they could from the galaxy center, where the local background is too luminous for our observations. Finally, there are eight bright confirmed clusters in our sample, all of which are located in the halo of the galaxy. These clusters lack spectroscopic observational data, especially for the metallicity measurements. Thus it is necessary to observe the spectra of our sample clusters systematically and constrain the spectroscopic metallicities and ages in detail.

The observational information of our sample GCs are listed in Table 1 which includes the names, coordinates, projected radii in kpc, exposures and observation dates. All the coordinates (R.A. and Dec. in Cols. 2 and 3) and projected radii from the galaxy center $r_{\mathrm{p}}$ (Col. 4) are all from RBC v.4, which were calculated with M31 center coordinate $00: 42: 44.31,+41: 16: 09.4$ (Perrett et al., 2002), $P A=38^{\circ}$ and distance $d=785 \mathrm{kpc}$ (McConnachie et al., 2005).

Table 1 The observations of our sample GCs.

\begin{tabular}{lccccc}
\hline ID & $\begin{array}{c}\text { R.A. } \\
(\mathrm{J} 2000)\end{array}$ & $\begin{array}{c}\text { Dec. } \\
(\mathrm{J} 2000)\end{array}$ & $\begin{array}{c}r_{p} \\
(\mathrm{kpc})\end{array}$ & $\begin{array}{c}\text { Exposure } \\
(\text { second })\end{array}$ & Date \\
\hline B289 & $00: 34: 20.882$ & $+41: 47: 51.14$ & 22.65 & 6000 & $08 / 28 / 2011$ \\
B290 & $00: 34: 20.947$ & $+41: 28: 18.18$ & 21.69 & 7200 & $09 / 01 / 2011$ \\
H11 & $00: 37: 28.028$ & $+44: 11: 26.41$ & 42.10 & 5400 & $09 / 01 / 2011$ \\
H18 & $00: 43: 36.030$ & $+44: 58: 59.30$ & 50.87 & 5400 & $08 / 29 / 2011$ \\
SK108A & $00: 47: 14.240$ & $+40: 38: 12.30$ & 14.47 & 3600 & $08 / 28 / 2011$ \\
SK112A & $00: 48: 15.870$ & $+41: 23: 31.20$ & 14.28 & 5400 & $08 / 29 / 2011$ \\
MGC1 & $00: 50: 42.459$ & $+32: 54: 58.78$ & 117.05 & 3600 & $08 / 28 / 2011$ \\
H25 & $00: 59: 34.560$ & $+44: 05: 39.10$ & 57.35 & 5400 & $09 / 01 / 2011$ \\
\hline
\end{tabular}

We show the spatial distribution of our sample eight halo GCs and all the confirmed GCs from RBC v.4 in Figure1 The large ellipse is the M31 disk/halo boundary as defined by Racine (1991). Note that all of our sample are located in the halo of M31, which can help us to access the nature of galaxy halo with an enlarged cluster sample, compared to Fan et al. (2011).

\section{OBSERVATIONS AND DATA REDUCTION}

Our Low-resolution spectroscopic observations were all taken at the $2.16 \mathrm{~m}$ optical telescope at Xinglong Site, which belongs to National Astronomical Observatories, Chinese Academy of Sciences (NAOC), from August 28th to September 1st, 2011 (Please see Table 11). An OMR (Optomechanics Research Inc.) spectrograph and a PI $1340 \times 400 \mathrm{CCD}$ detector were used during this run with a dispersion of 200 $\AA \mathrm{mm}^{-1}, 4.8 \AA \mathrm{pixel}^{-1}$, and a $3.0^{\prime \prime}$ slit. The typical seeing there was $\sim 2.5^{\prime \prime}$. The spectra cover the wavelength range of $3500-8100 \AA$ at $4 \AA$ resolution. All our spectra have $S / N \geq 40$.

In order to calibrate our $2.16 \mathrm{~m}$ data onto the Lick system, we also observed eleven Lick standard stars (HR 6806, HR 6815, HR 7030, HR 7148, HR 7171, HR 7503, HR 7504, HR 7576, HR 7977, HR 8020, HR 8165) near our field, which are selected from a catalogue of all 25 index measurements and coordinates for 460 stars (ref, available from http://astro.wsu.edu/worthey/html/system.html; Worthey \& Ottaviani, 1997; Worthey et al., 1994a). Most of these standard stars are luminous $(\sim 5-6$ in $\mathrm{V}$ band), hence the exposure time we took was 20 second with the OMR system.

The spectroscopic data were reduced following the standard procedures with NOAO Image Reduction and Analysis Facility (IRAF v.2.15) software package. First, the spectra have been bias and flat-field corrected, as well as cosmic-ray removed. Then the wavelength calibrations were performed based on Helium/Argon lamps exposed at both the beginning and the end of the observations in each night. Flux calibrations were performed based on observations of at least two of the KPNO spectral standard stars (Massey et al., 1988) each night. The atmospheric extinction was corrected with the mean extinction coefficients measurements of Xinglong through the Beijing-Arizona-Taiwan-Connecticut (BATC) multicolor sky survey (H. J. Yan 1995, priv. comm.).

Before the Lick indices were measured, the heliocentric radial velocities $V_{r}$ were measured by comparing the absorption lines of our spectra with the templates in various radial velocities. The typical 


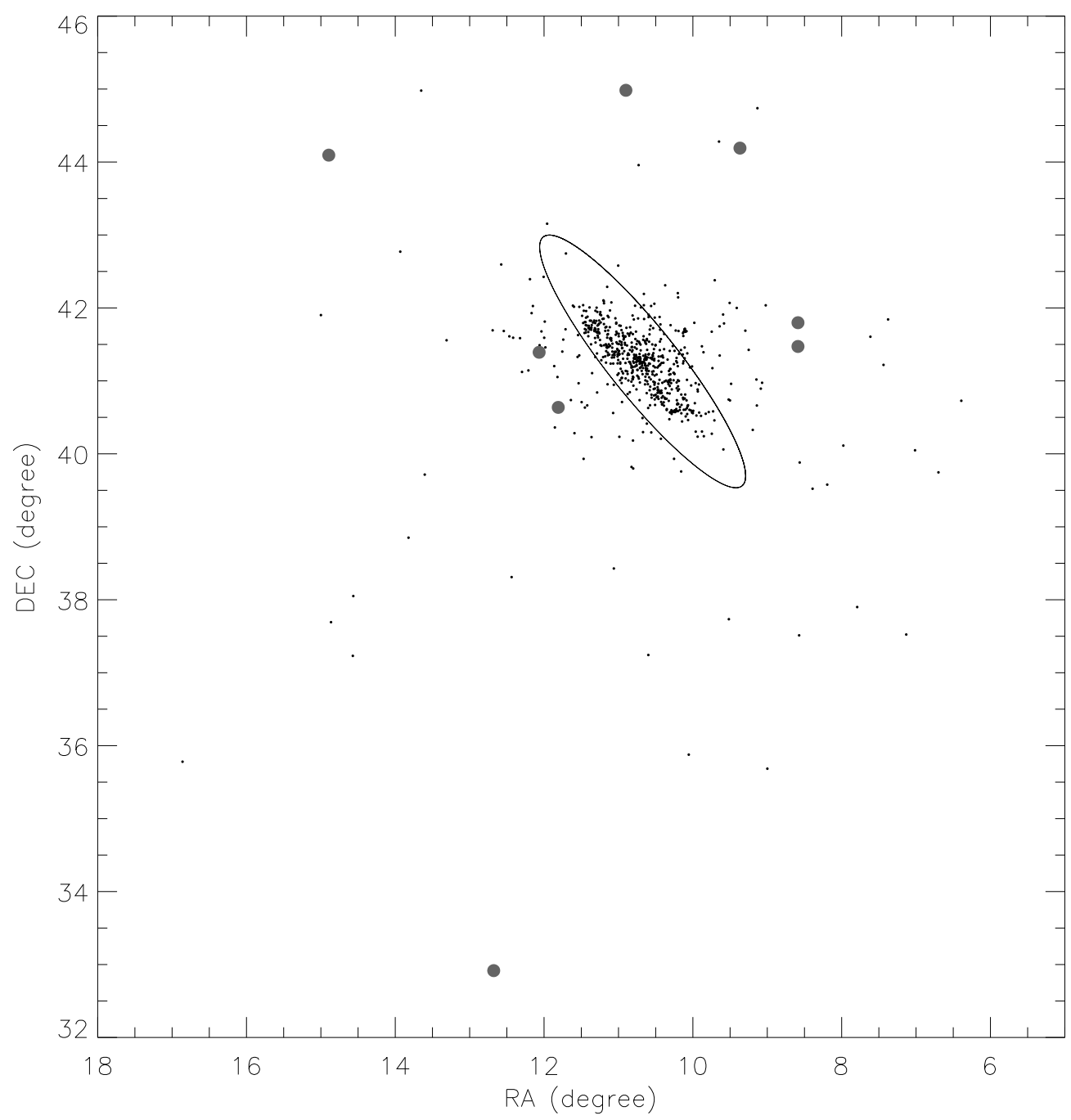

Fig. 1 Spatial distribution of M31 GCs. Our sample halo GCs are shown with filled circles and the confirmed GCs from RBC v.4 are marked with points. The large ellipse is the M31 disk/halo boundary as defined in Racine (1991).

internal velocity errors on a single measure is $\sim 20 \mathrm{~km} \mathrm{~s}^{-1}$. The estimated radial velocities $V_{r}$ with the associated uncertainties (Col. 2) are listed in Table 2 The published radial velocities $V_{r}(\mathrm{Col} .3)$ are also listed for comparisons. The systematic difference between our observed velocity and the catalogue velocity is found to be $29 \pm 39 \mathrm{~km} \mathrm{~s}^{-1}$ and the standard deviation of the differences between our observed velocity and the catalogue velocity is $78 \mathrm{~km} \mathrm{~s}^{-1}$ for the five pairs of the radial velocities. It suggests that our measurements agree with those listed in RBC v.4 since the systematic difference between our measurements and the published values is not significant.

Figure 2 shows the radial velocity $V_{r}$ (corrected for the systemic velocity of M31) as a function of the projected radii from the galaxy center. The Left panel is for the all confirmed GCs which have 

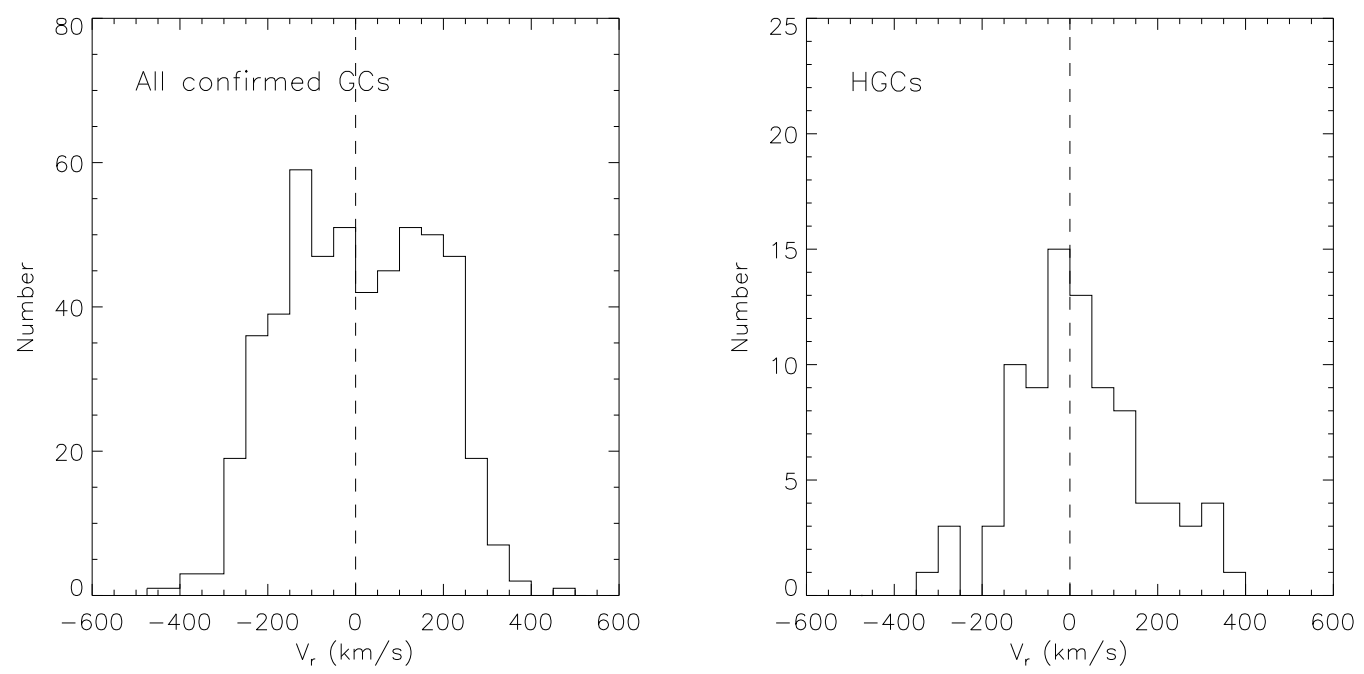

Fig. 2 The distributions of radial velocity $V_{r}$ (corrected for the systemic velocity of M31). Left: all the confirmed GCs. Right: the HGCs only.

the radial velocity $V_{r}$ measurements and the Right panel is for the HGCs, which refers to the GCs in the galaxy halo defined in Figure 1 It can be noted that the radial velocity distributions are basically symmetric in distributions either for all the confirmed GC sample or for the HGCs only.

Table 2 The radial velocities $V_{r}$ of our sample GCs as well as the previous results.

\begin{tabular}{lcc}
\hline ID & our work & RBC v.4 \\
\hline B289 & $-96.81 \pm 47.27$ & $-181 \pm 30$ \\
B290 & $-488.73 \pm 43.14$ & $-381 \pm 26$ \\
H11 & $-173.02 \pm 39.63$ & \\
H18 & $-300.48 \pm 79.65$ & \\
SK108A & $-352.17 \pm 19.18$ & $-379 \pm 38$ \\
SK112A & $-342.68 \pm 32.81$ & $-252 \pm 46$ \\
MGC1 & $-412.67 \pm 17.13$ & $-355 \pm 2$ \\
H25 & $-256.49 \pm 55.28$ & \\
\hline
\end{tabular}

We plotted the radial velocities $V_{r}$ versus the projected radii $r_{p}$ in Figure 3 where the radial velocities have been corrected for the systemic velocity of M31 galaxy of $300 \pm 4 \mathrm{~km} \mathrm{~s}^{-1}$ (Perrett et al., 2002). The Left panel is for all the confirmed clusters in RBC v.4 while the right panel is for the halo clusters which are defined in Figure 1. The points are the published measurements from RBC v.4 while the open triangles and the filled circles with errors are the measurements in Paper I and those in our work, respectively. In the Right panel, the symbols are the same as those in the Left panel. We find that the dispersion of the velocity becomes smaller when the GCs are locate further from the center of the galaxy with larger projected radius $r_{p}$. It can be seen that the dispersion of the radial velocity becomes smaller when the projected radius $r_{p}$ is larger.

Subsequently, all the spectra were shifted to the zero radial velocity and smoothed to the wavelength dependent Lick resolution with a variable-width Gaussian kernel following the definition of Worthey \& Ottaviani (1997), i.e. $11.5 \AA$ at $4000 \AA, 9.2 \AA$ at $4400 \AA, 8.4 \AA$ at $4900 \AA, 8.4 \AA$ at 5400 

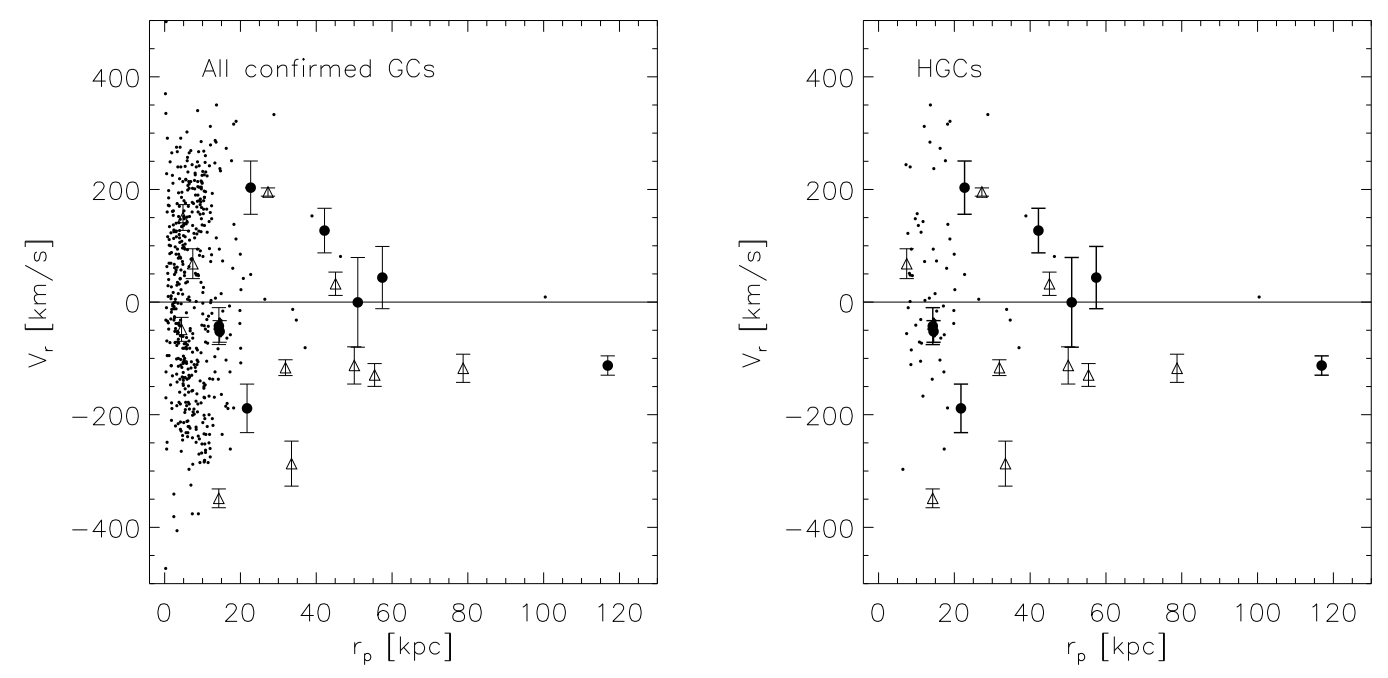

Fig. 3 The radial velocity $V_{r}$ (corrected for the systemic velocity of M31) as a function of the projected radius. Left: all confirmed clusters and Right: the halo clusters. The filled circles with errors are the halo GCs from our sample while the points represent the velocities from RBC v.4 catalogue.

$\AA, 9.8 \AA$ at $6000 \AA$. Indeed, we measured all the 25 types of Lick indices strictly by using the parameters and formulae from Worthey et al. (1994a) and Worthey \& Ottaviani (1997). The uncertainty of each index was estimated based on the analytic formulae (11)-(18) of Cardiel et al. (1998).

Eq.11 is the linear fit formula for calibrating the raw measurements of our $2.16 \mathrm{~m}$ data to the standard Lick index system. The eleven standard stars are utilized for the fitting (Please see Figure 4t) and the results are listed in Table 3

$$
\mathrm{EW}_{\text {ref }}=a+b \cdot \mathrm{EW}_{\text {raw }}
$$

\section{FITTING, ANALYSIS AND RESULTS}

\subsection{Model description}

Thomas et al. (2003) provided stellar population models including Lick absorption line indices for various elemental-abundance ratios, covering ages from 1 to $15 \mathrm{Gyr}$ and metallicities from 1/200 to $3.5 \times$ solar abundance. These models are based on the standard models of Maraston (1998), with input stellar evolutionary tracks from Cassisi et al. (1997) and Bono et al. (1997) and a Salpeter (1955) stellar initial mass function. Thomas et al. (2004) improved the models by including higher-order Balmer absorptionline indices. They found that these Balmer indices are very sensitive to changes in the $\alpha / \mathrm{Fe}$ ratio for supersolar metallicities. The latest stellar population model for Lick absorption-line indices (Thomas et al., 2010) is an improvement on Thomas et al. (2003) and Thomas et al. (2004). They were derived from the MILES stellar library, which provides a higher spectral resolution appropriate for MILES and SDSS spectroscopy, as well as flux calibration. The models cover ages from 0.1 to $15 \mathrm{Gyr},[\mathrm{Z} / \mathrm{H}]$ from -2.25 to $0.67 \mathrm{dex}$, and $[\alpha / \mathrm{Fe}]$ from -0.3 to $0.5 \mathrm{dex}$. In our work, we fitted our absorption indices based on the models of Thomas et al. (2010), by using the two sets of stellar evolutionary tracks provided, i.e., Cassisi et al. (1997) and Padova. 

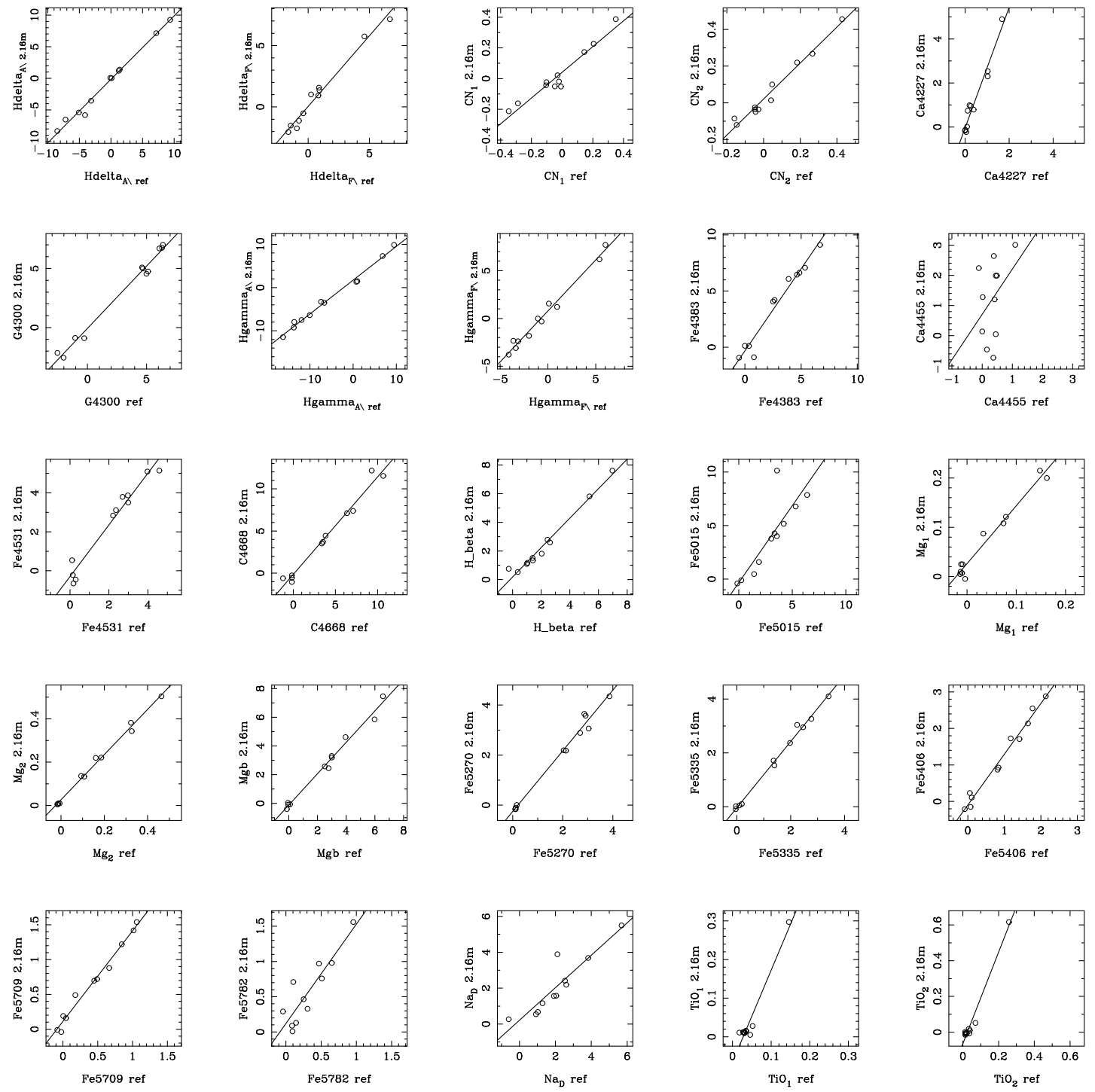

Fig. 4 Calibrations of index measurements from the eleven standard stars of $2.16 \mathrm{~m}$ raw spectra with those from reference Worthey \& Ottaviani (1997); Worthey et al. (1994a). The linear fit coefficients of Eq. 3 have been derived to be used for calibrating our raw data to the Lick index system.

\subsection{Fits with stellar population models and the results}

Similar to Sharina et al. (2006) and our Paper I, the $\chi^{2}-$ minimization routine was applied for fitting Lick indices with the SSP models to derive the physical parameters. As we measured 25 different types of Lick line indices listed in Table 3 , all indices were used for the fitting procedure. As Thomas et al. (2010) provide only 20 ages, 6 metallicity $[\mathrm{Z} / \mathrm{H}]$, and $4 \alpha$-element $[\alpha / \mathrm{Fe}]$ for the SSP model, it is necessary to interpolate the original models to the higher-resolution models for our needs. We performed the cubic spline interpolations, using equal step lengths, to obtain a grid of 150 ages from 0.1 to $15 \mathrm{Gyr}, 31$ 
Table 3 The Linear Fit Coefficients $a$ and $b$ in Eq. 3 for transformations of the $2.16 \mathrm{~m}$ data to the Lick index system.

\begin{tabular}{lcc}
\hline Index & $a$ & $b$ \\
\hline $\mathrm{H} \delta_{\mathrm{A}}(\AA)$ & $-0.15 \pm 0.19$ & $1.00 \pm 0.04$ \\
$\mathrm{H} \delta_{\mathrm{F}}(\AA)$ & $0.04 \pm 0.15$ & $1.15 \pm 0.06$ \\
$\mathrm{CN} 1(\mathrm{mag})$ & $0.04 \pm 0.01$ & $0.84 \pm 0.07$ \\
$\mathrm{CN} 2(\mathrm{mag})$ & $0.02 \pm 0.01$ & $0.98 \pm 0.05$ \\
$\mathrm{Ca} 4227(\AA)$ & $-0.04 \pm 0.14$ & $2.73 \pm 0.21$ \\
$\mathrm{G} 4300(\AA)$ & $-0.06 \pm 0.19$ & $1.05 \pm 0.04$ \\
$\mathrm{H} \gamma_{\mathrm{A}}(\AA)$ & $1.73 \pm 0.26$ & $0.78 \pm 0.03$ \\
$\mathrm{H} \gamma_{\mathrm{F}}(\AA)$ & $0.79 \pm 0.16$ & $1.07 \pm 0.05$ \\
$\mathrm{Fe} 3383(\AA)$ & $-0.32 \pm 0.36$ & $1.46 \pm 0.10$ \\
$\mathrm{Ca} 4455(\AA)$ & $0.71 \pm 0.56$ & $1.50 \pm 1.21$ \\
$\mathrm{Fe} 4531(\AA)$ & $-0.30 \pm 0.24$ & $1.33 \pm 0.09$ \\
$\mathrm{Fe} 4668(\AA)$ & $-0.16 \pm 0.31$ & $1.16 \pm 0.06$ \\
$\mathrm{H} \beta(\AA)$ & $0.17 \pm 0.16$ & $1.03 \pm 0.05$ \\
$\mathrm{Fe} 5015(\AA)$ & $-0.34 \pm 1.07$ & $1.44 \pm 0.30$ \\
$\mathrm{Mg} 1(\mathrm{mag})$ & $0.03 \pm 0.01$ & $1.18 \pm 0.07$ \\
$\mathrm{Mg} 2(\mathrm{mag})$ & $0.03 \pm 0.01$ & $1.04 \pm 0.03$ \\
$\mathrm{Mg} b(\AA)$ & $-0.12 \pm 0.15$ & $1.09 \pm 0.04$ \\
$\mathrm{Fe} 5270(\AA)$ & $-0.25 \pm 0.11$ & $1.21 \pm 0.05$ \\
$\mathrm{Fe} 5335(\AA)$ & $-0.04 \pm 0.06$ & $1.23 \pm 0.03$ \\
$\mathrm{Fe} 5406(\AA)$ & $-0.10 \pm 0.08$ & $1.39 \pm 0.07$ \\
$\mathrm{Fe} 5709(\AA)$ & $0.11 \pm 0.03$ & $1.30 \pm 0.06$ \\
$\mathrm{Fe} 5782(\AA)$ & $0.12 \pm 0.10$ & $1.41 \pm 0.24$ \\
$\mathrm{NaD}(\AA)$ & $0.21 \pm 0.36$ & $0.91 \pm 0.14$ \\
$\mathrm{TiO} 1(\mathrm{mag})$ & $-0.07 \pm 0.01$ & $2.38 \pm 0.19$ \\
$\mathrm{TiO} 2(\mathrm{mag})$ & $-0.07 \pm 0.01$ & $2.56 \pm 0.14$ \\
\hline
\end{tabular}

$[\mathrm{Z} / \mathrm{H}]$ values from -2.25 to $0.67 \mathrm{dex}$, and $51[\alpha / \mathrm{Fe}]$ from -0.3 to $0.5 \mathrm{dex}$, which could make the fitted results smoother and more continuous. Since Worthey (1994b); Galleti et al. (2009) pointed out the agemetallicity degenaracy for most of the spectral feature indices measurements, which almost remain the same when the percentage change $\Delta$ age $/ \Delta Z=3 / 2$. Therefore, it is necessary for us to constrain the metallicity with the metal-sensitive indices before the fits.

Fortunately, Galleti et al. (2009) provide two ways to measure the metallicity from the metalsensitive spectral indices directly. One method is through combining the absorption line indices $\mathrm{Mg}$ and $\mathrm{Fe},[\mathrm{MgFe}]$, which is defined as $[\mathrm{MgFe}]=\sqrt{\mathrm{Mg} b \cdot\langle\mathrm{Fe}\rangle}$, where $\langle\mathrm{Fe}\rangle=(\mathrm{Fe} 5270+\mathrm{Fe} 5335) / 2$. Thus, the metallicity can be calculated from the formula below,

$$
[\mathrm{Fe} / \mathrm{H}]_{[\mathrm{MgFe}]}=-2.563+1.119[\mathrm{MgFe}]-0.106[\mathrm{MgFe}]^{2} \pm 0.15
$$

The second way to obtain the metallicity from $\mathrm{Mg} 2$ is using a polynomial in the following,

$$
[\mathrm{Fe} / \mathrm{H}]_{\mathrm{Mg} 2}=-2.276+13.053 \mathrm{Mg} 2-16.462 \mathrm{Mg} 2^{2} \pm 0.15 .
$$

Finally we obtained $[\mathrm{Fe} / \mathrm{H}]_{\text {avg }}$ with uncertainty in Table 4 , which is an average of the metallicities derived from the metallicity Eqs. 2 and 3 , respectively. The averaged metallicity $[\mathrm{Fe} / \mathrm{H}]_{\text {avg }}$ will be used to constrain the metallicity in the fits to break the age-metallicity trends/degeneracy. However, Thomas et al. (2010) model only provide the metallicity parameters with $[\mathrm{Z} / \mathrm{H}]$ and $[\alpha / \mathrm{Fe}]$, thus we need to find a relationship between the iron abundance $[\mathrm{Fe} / \mathrm{H}]$, total metallicity $[\mathrm{Z} / \mathrm{H}]$ and $\alpha$-element to iron ratio $[\alpha / \mathrm{Fe}]$, which we can replace $[\mathrm{Fe} / \mathrm{H}]$ with $[\mathrm{Z} / \mathrm{H}]$ and $[\alpha / \mathrm{Fe}]$ in the fit procedure. In fact, Thomas et al. (2003) give the relation in Eq.4

$$
[\mathrm{Z} / \mathrm{H}]=[\mathrm{Fe} / \mathrm{H}]+0.94[\alpha / \mathrm{Fe}]
$$


Table 4 The metallicities $[\mathrm{Fe} / \mathrm{H}]$ derived from the spectral indices $[\mathrm{MgFe}], \mathrm{Mg} 2$.

\begin{tabular}{cc}
\hline Name & {$[\mathrm{Fe} / \mathrm{H}]_{\mathrm{avg}}$} \\
\hline B289 & $-1.83 \pm 0.27$ \\
B290 & $-0.56 \pm 0.63$ \\
H11 & $-0.49 \pm 0.58$ \\
H18 & $-1.35 \pm 0.65$ \\
SK108A & $-2.35 \pm 0.22$ \\
SK112A & $-1.62 \pm 0.43$ \\
MGC1 & $-2.06 \pm 0.33$ \\
H25 & $-2.74 \pm 0.47$ \\
\hline
\end{tabular}

Notes: Here we define $[\mathrm{Fe} / \mathrm{H}]_{\mathrm{avg}}=\frac{[\mathrm{Fe} / \mathrm{H}]_{[\mathrm{MgFe}]}+[\mathrm{Fe} / \mathrm{H}]_{\mathrm{Mg} 2}}{2}$

Here we would like to draw reader's attention that although the metallicity $[\mathrm{Fe} / \mathrm{H}]$ has been determined primarily, there are still many different ways to combine $[\mathrm{Z} / \mathrm{H}]$ and $[\alpha / \mathrm{Fe}]$ in the parameter grid of the model. Therefore, we still need to fit the age, $[\mathrm{Z} / \mathrm{H}]$ and $[\alpha / \mathrm{Fe}]$ simultaneously. Here, we would like to constrain the metallicity in the fits for $\left|[\mathrm{Fe} / \mathrm{H}]_{\text {fit }}-[\mathrm{Fe} / \mathrm{H}]_{\text {avg }}\right| \leq 0.3$ dex, which is the typical metallicity uncertainty for the observations and it will make the fits more reasonable. Like the Paper I, the physical parameters ages, metallicities $[\mathrm{Z} / \mathrm{H}]$, and $[\alpha / \mathrm{Fe}]$ can be determined by comparing the interpolated stellar population models with the observational spectral feature indices by employing the $\chi^{2}$-minimization method below,

$$
\chi_{\min }^{2}=\min \left[\sum_{i=1}^{25}\left(\frac{L_{\lambda_{i}}^{\text {obs }}-L_{\lambda_{i}}^{\text {model }}(\text { age },[\mathrm{Z} / \mathrm{H}],[\alpha / \mathrm{Fe}])}{\sigma_{i}}\right)^{2}\right],
$$

where $L_{\lambda_{i}}^{\text {model }}$ (age, $\left.[\mathrm{Z} / \mathrm{H}],[\alpha / \mathrm{Fe}]\right)$ is the $i^{\text {th }}$ Lick line index in the stellar population model for age, metallicity $[\mathrm{Z} / \mathrm{H}]$, and $[\alpha / \mathrm{Fe}]$, while $L_{\lambda_{i}}^{\text {obs }}$ represents the observed calibrated Lick absorption-line indices from our measurements and the errors estimated in our fitting are given as follows,

$$
\sigma_{i}^{2}=\sigma_{\mathrm{obs}, i}^{2}+\sigma_{\text {model }, i}^{2} .
$$

Here, $\sigma_{\text {obs. } i}$ is the observational uncertainty while $\sigma_{\text {model }, i}$ is the uncertainty associated with the models of Thomas et al. (2010). These two types of uncertainties have been both considered in our fitting procedure.

Table 5 lists the fitted ages, $[\mathrm{Z} / \mathrm{H}]$ and $[\alpha / \mathrm{Fe}]$ with different evolutionary tracks of Cassisi et al. (1997) and Padova, respectively. In addition, we calculated the $[\mathrm{Fe} / \mathrm{H}]_{\text {cassisi }}$ and $[\mathrm{Fe} / \mathrm{H}]_{\text {padova }}$ by applying the Eq. 4 to the fitted $[\mathrm{Z} / \mathrm{H}]$ and $[\alpha / \mathrm{Fe}]$. For the reason of keeping consistency with Paper I, we adopted the metallicity $[\mathrm{Fe} / \mathrm{H}]_{\text {cassisi }}$ in the following statistics and analysis. From Table 5, we found that the ages, $[\mathrm{Z} / \mathrm{H}]$ and the $\alpha$-element $[\alpha / \mathrm{Fe}]$ fitted from either Cassisi et al. (1997) or Padova tracks are consistant with each other. Besides, it is worth noting that all of our sample halo GCs are older than $10 \mathrm{Gyr}$ in both evolutionary tracks except B290 (5.5 to $5.8 \mathrm{Gyr}$ ), which is older than $2 \mathrm{Gyr}$ and it should be identified as the "old" in Caldwell et al. (2009). Thus, it indicates that these halo clusters formed at the early stage of the galaxy formation, which agrees well with the previous findings.

Actually, Mackey et al. (2010) conclude that the metal abundance of MGC1 is about $[\mathrm{Fe} / \mathrm{H}]=-2.3$ and age is 12.5 to $12.7 \mathrm{Gyr}$ through the color-magnitude diagram fitting. The age estimated agree well with our results while the metallicity is lower than our estimate $[\mathrm{Fe} / \mathrm{H}]_{\mathrm{avg}}=-2.06 \pm 0.33$ in Table 4 or $[\mathrm{Fe} / \mathrm{H}]_{\text {cassis }}=-1.76 \pm 0.16$ in Table 5. Nevertheless, Alves-Brito et al. (2009) found that the metallicity $[\mathrm{Fe} / \mathrm{H}]=-1.37 \pm 0.15$ by combining the spectroscopic data and the photometric data, which is higher than our estimate. Hence, it can be seen that our result is just between the two results, suggesting that our result agrees with the previous conclusions.

\subsection{Metallicity Properties of Outer Halo}

The metallicity gradient of the halo star clusters and stars are important to the formation and enrichment processes of their host galaxy. Basically, there are two possible scenarios for the galaxy formation. 
Table 5 The $\chi^{2}$-minimization Fitting Results Using Thomas et al. (2010) Models with Cassisi et al. (1997) and Padova Stellar Evolutionary Tracks, respectively.

\begin{tabular}{ccccccccc}
\hline \multicolumn{1}{c}{ Cassisi } & \multicolumn{3}{c}{ Padova } \\
\hline Name & $\begin{array}{c}\text { Age } \\
(\mathrm{Gyr})\end{array}$ & $\begin{array}{c}{[\mathrm{Z} / \mathrm{H}]} \\
(\mathrm{dex})\end{array}$ & $\begin{array}{c}{[\alpha / \mathrm{Fe}]} \\
(\mathrm{dex})\end{array}$ & $\begin{array}{c}{[\mathrm{Fe} / \mathrm{H}]} \\
(\mathrm{dex})\end{array}$ & $\begin{array}{c}\text { Age } \\
(\mathrm{Gyr})\end{array}$ & $\begin{array}{c}{[\mathrm{Z} / \mathrm{H}]} \\
(\mathrm{dex})\end{array}$ & $\begin{array}{c}{[\alpha / \mathrm{Fe}]} \\
(\mathrm{dex})\end{array}$ & $\begin{array}{c}{[\mathrm{Fe} / \mathrm{H}]} \\
(\mathrm{dex})\end{array}$ \\
\hline B289 & $10.75 \pm 4.15$ & $-1.67 \pm 0.23$ & $0.34 \pm 0.16$ & $-2.09 \pm 0.27$ & $11.70 \pm 2.80$ & $-2.07 \pm 0.18$ & $-0.12 \pm 0.18$ & $-2.13 \pm 0.25$ \\
B290 & $5.80 \pm 2.40$ & $-0.99 \pm 0.05$ & $-0.26 \pm 0.05$ & $-0.85 \pm 0.07$ & $5.50 \pm 0.40$ & $-1.33 \pm 0.38$ & $-0.26 \pm 0.05$ & $-0.85 \pm 0.39$ \\
H11 & $13.75 \pm 1.25$ & $0.09 \pm 0.32$ & $0.08 \pm 0.05$ & $-0.19 \pm 0.33$ & $13.60 \pm 0.20$ & $-0.10 \pm 0.24$ & $0.00 \pm 0.06$ & $-0.21 \pm 0.24$ \\
H18 & $13.45 \pm 1.45$ & $-0.47 \pm 0.37$ & $0.48 \pm 0.02$ & $-1.07 \pm 0.37$ & $13.60 \pm 0.20$ & $-0.50 \pm 0.24$ & $0.48 \pm 0.02$ & $-1.07 \pm 0.24$ \\
SK108A & $13.60 \pm 0.30$ & $-1.53 \pm 0.18$ & $0.28 \pm 0.22$ & $-2.09 \pm 0.28$ & $13.55 \pm 0.45$ & $-1.48 \pm 0.23$ & $0.27 \pm 0.24$ & $-2.09 \pm 0.32$ \\
SK112A & $11.10 \pm 3.90$ & $-1.33 \pm 0.38$ & $0.25 \pm 0.25$ & $-1.35 \pm 0.45$ & $11.70 \pm 3.30$ & $-1.51 \pm 0.47$ & $0.10 \pm 0.40$ & $-1.42 \pm 0.61$ \\
MGC1 & $13.30 \pm 0.80$ & $-1.39 \pm 0.14$ & $0.42 \pm 0.08$ & $-1.76 \pm 0.16$ & $12.90 \pm 1.30$ & $-1.39 \pm 0.14$ & $0.42 \pm 0.08$ & $-1.76 \pm 0.16$ \\
H25 & $13.60 \pm 0.30$ & $-1.98 \pm 0.20$ & $0.50 \pm 0.00$ & $-2.45 \pm 0.20$ & $13.50 \pm 0.50$ & $-2.03 \pm 0.05$ & $0.50 \pm 0.00$ & $-2.45 \pm 0.05$ \\
\hline
\end{tabular}

One is that the halo stars and clusters should feature large-scale metallicity gradients if the enrichment timescale is shorter than the collapse time, which may be due to the galaxy formation as a consequence of a monolithic, dissipative, and rapid collapse of a single massive, nearly spherical, spinning gas cloud (Eggen et al., 1962; Barmby et al., 2000). The other one is a chaotic scheme for early galactic evolution, when the loosely bound pre-enriched fragments merge with the protogalaxy during a very long period of time, in which case a more homogeneous metallicity distribution should develop (Searle \& Zinn, 1978). However, most galaxies are believed to have formed through a combination of these scenarios.

van den Bergh (1969); Huchra et al. (1982) showed that there is little or no evidence for a general radial metallicity gradient for GCs within a radius of 50 arcmin. However, studies including Huchra et al. (1991); Perrett et al. (2002); Fan et al. (2008) support the possible existence of a radial metallicity gradient for the metal-poor M31 GCs, although the slope is not very significant. Perrett et al. (2002) suggest that the gradients is -0.017 and $-0.015 \mathrm{dex} \operatorname{arcmin}^{-1}$ for the full sample and inner metal-poor clusters. More recently, Fan et al. (2008) found that the slope is -0.006 and -0.007 dex $\operatorname{arcmin}^{-1}$ for the metal-poor subsample and whole sample while the slope approaches zero for the metal-rich subsample. Nevertheless, all these studies are based on GCs that are located relatively close to the center of the galaxy, usually at projected radii of less than 100 arcmin. Recently, Huxor et al. (2011) investigated the metallicity gradient for 15 halo GCs to $r_{\mathrm{p}}=117 \mathrm{kpc}$ with the metallicity derived from the CMD fittings Mackey et al. (2006, 2007, 2010) and the authors found that the metallicity gradient becomes insignificant if one halo GC H14 is excluded in their Figure 6. We found that our result is consistent well with the previous findings of Huxor et al. (2011). In Paper I, we found the slope of metallicity gradient is $-0.018 \pm 0.001 \mathrm{dex} \mathrm{kpc}^{-1}$ for the halo clusters sample extended to $r_{\mathrm{p}} \sim 117 \mathrm{kpc}$ from the galaxy center. Further, the slope turns to be $-0.010 \pm 0.002 \mathrm{dex} \mathrm{kpc}{ }^{-1}$ if only considering the clusters $r_{p}>25$ $\mathrm{kpc}$.

Since we have spectroscopic observations of eight more halo confirmed clusters, it is interesting to check if the metallicity distribution/spatial gradient would change with an enlarged halo clusters sample. For the new observed data, as we recalled in 4.2 , only MGC1 have the previous metallicity measurements from the literatures, which are very different for different works and our measurement is just the median value. Thus, we adopted our measurement. Finally we have a metallicity sample of 391 entries in total.

Figure 5 shows the metallicity as a function of projected radius from the galaxy center for all outer GCs with spectroscopic metallicity with $r_{\mathrm{p}}>25 \mathrm{kpc}$ from the galaxy center. The slope of a linear fit is $-0.005 \pm 0.005 \mathrm{dex} \mathrm{kpc}^{-1}$, which is marked with a solid black line. However, if the two highest metallicity star clusters G001 and H11 are excluded, the slope turns out to be $-0.002 \pm 0.003$ dex $\mathrm{kpc}^{-1}$, which is shown with the red dashed line. Thus, both of the cases suggest there is none metallicity gradient for the M31 outer halo clusters when $r_{\mathrm{p}}>25 \mathrm{kpc}$, which agree with the conclusion of Paper I. 


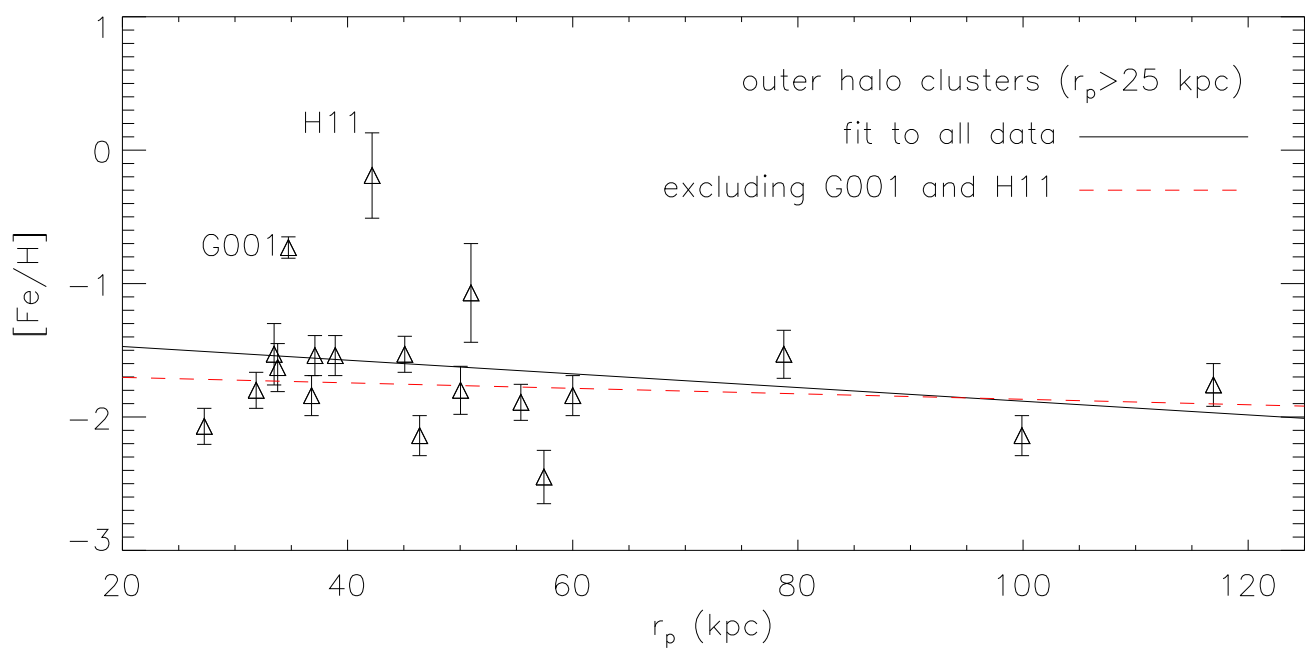

Fig. 5 Metallicities $[\mathrm{Fe} / \mathrm{H}]$ versus projected radii for the outer halo GCs with $r_{\mathrm{p}}>25 \mathrm{kpc}$ from the center of the galaxy. The slope of the linear fitting is $-0.005 \pm 0.005 \mathrm{dex} \mathrm{kpc}^{-1}$ (black solid line). However, if the two highest metallicity GCs G001 and H11 are excluded, the slope turns out to be $-0.002 \pm 0.003 \mathrm{dex} \mathrm{kpc}^{-1}$ (red dashed line).

Table 6 The slopes of metallicity gradient by adding the random errors to the data.

\begin{tabular}{ccc}
\hline No. & $k_{\text {all }}$ & $k_{<-1}$ \\
\hline 1 & $-0.013 \pm 0.010$ & $-0.013 \pm 0.011$ \\
2 & $-0.003 \pm 0.010$ & $0.000 \pm 0.014$ \\
3 & $-0.011 \pm 0.012$ & $-0.008 \pm 0.012$ \\
4 & $-0.009 \pm 0.009$ & $0.000 \pm 0.012$ \\
5 & $-0.003 \pm 0.013$ & $-0.036 \pm 0.021$ \\
6 & $-0.002 \pm 0.012$ & $0.004 \pm 0.022$ \\
7 & $-0.004 \pm 0.013$ & $-0.002 \pm 0.022$ \\
8 & $-0.009 \pm 0.012$ & $0.009 \pm 0.015$ \\
9 & $-0.005 \pm 0.010$ & $-0.008 \pm 0.017$ \\
10 & $-0.013 \pm 0.011$ & $0.003 \pm 0.008$ \\
\hline
\end{tabular}

Therefore it seems that the "fragments merging" scenario dominated in the outer halo during the galaxy formation stage.

It should be noted that the metallicity gradient is fitted based on the data of our observations and the literature and the metallicities from different literature may not the same. For instance, the metallicity of $\mathrm{G} 001$ is $[\mathrm{Fe} / \mathrm{H}]=-1.08 \pm 0.09$ in Huchra et al. (1991) while $[\mathrm{Fe} / \mathrm{H}]=-0.73 \pm 0.15$ in Galleti et al. (2009). Thus we wonder how the slope would change when the data is changed. We simulated ten sets of random data from $\sigma=-0.5$ to 0.5 and added them to the metallicities that we used in Figure 5 and then refit the slopes again for ten times separatedly and the results are shown in Table 6 It shows that the slope dose not change significantly when the simulated errors were added, suggesting that the slope is stable even the data from different measures. 


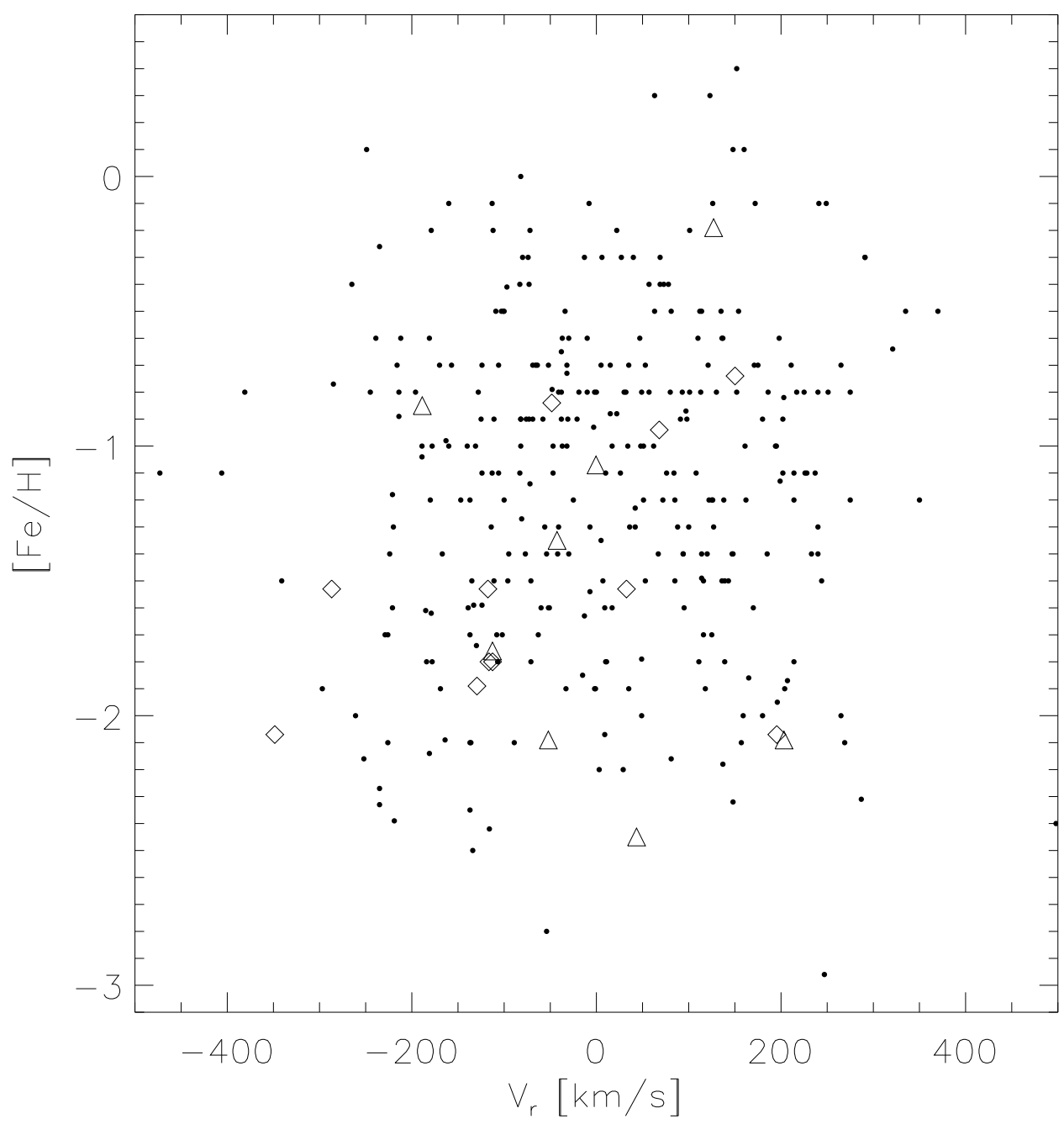

Fig. 6 Metallicity $[\mathrm{Fe} / \mathrm{H}]$ versus radial velocity $V_{r}$ (corrected for the systemic velocity of M31) for all the GCs with spectroscopic metallicities and radial velocity. The small points are from the literature; the squares are from Paper I; the triangles are from our measurement.

Figure 6 shows the relationship between the metallicities and the radial velocities $V_{r}$ which have been corrected for the systemic velocity of the M31 galaxy. The spectroscopic metallicities are from the literature (Huchra et al., 1991; Barmby et al., 2000; Perrett et al., 2002; Galleti et al., 2009; Caldwell et al., 2011), Paper I as well as this work and the radial velocities $V_{r}$ are from the RBC v.4, Paper I and this work. It seems that there is no any relationship between the metallicities versus the radial velocities $V_{r}$.

Figure 7 shows the metallicities versus ages of the GCs. The metallicities are from the literature (Huchra et al., 1991; Barmby et al., 2000; Perrett et al., 2002; Galleti et al., 2009; Caldwell et al., 2011), Paper I as well as this work and the ages are from the Fan et al. (2010), Paper I and this work. We 


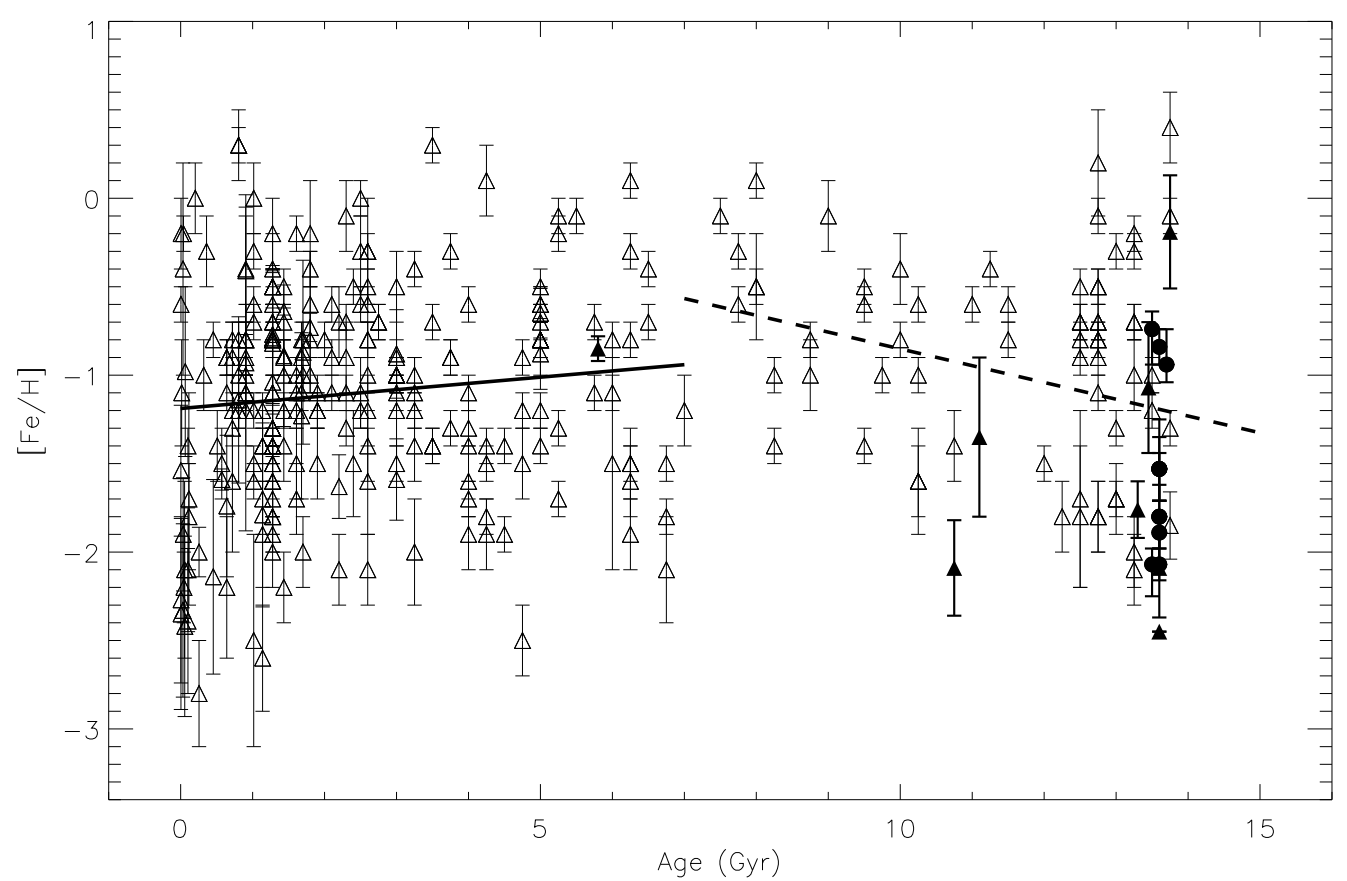

Fig. 7 Metallicity $[\mathrm{Fe} / \mathrm{H}]$ versus ages for all the clusters with spectroscopic metallicity and age estimates. The open triangles are the data from the literature; the filled circles are the data from Paper I; the filled triangles are the data from this work. The solid line represents the linear fit of GCs younger than $7 \mathrm{Gyr}$ while the dashed line is the fit for the GCs older than 7 Gyr.

would like to see if there is any relationship between the ages and metallicities for these GCs. Actually we find that the relationships are different for the GC populations with different age. The slope of the GCs younger than $7 \mathrm{Gyr}$ is $k=0.035 \pm 0.021$ while the slope of the GCs older than $7 \mathrm{Gyr}$ is $k=-0.095 \pm 0.034$, which is $\sim 3 \sigma$ significant level. It suggests that for the GCs younger than $7 \mathrm{Gyr}$, there is no relationship between the age and metallicity while for the clusters older than $7 \mathrm{Gyr}$, it seems that the older GCs are more metal-poor (lower metallicity) and the younger GCs are more metal-rich (higher metallicity).

Previously, many astronomers found the significant bimodal case in the metallicity of M31 GC distribution by applying the mixture-model KMM test (Ashman et al., 1994). Ashman \& Bird (1993); Barmby et al. (2000); Perrett et al. (2002) found the proportion of the metal-poor and metal-rich group is $\sim 2: 1$ to $\sim 3: 1$ with the peak positions of $[\mathrm{Fe} / \mathrm{H}] \approx-1.5$ and -0.6 , respectively. Fan et al. (2008) examined the bimodality of metallicity distribution with a larger sample and the authors found the proportion is $\sim 1.5: 1$ and the the peak positions are $[\mathrm{Fe} / \mathrm{H}] \sim-1.7$ and $\sim-0.7$, respectively. However, the recent work of Caldwell et al. (2011) suggests that there is no significant bimodality or trimodality for metallicity distribution with a sample of $322 \mathrm{M} 31 \mathrm{GCs}$, most of which have spectroscopic metallicity with high $\mathrm{S} / \mathrm{N}$ ratio. Since we have new observation data and a larger spectroscopic data sample, we are able to reexamine the bimodality of the metallicity distributions of M31 GCs. Figure 8 shows the metallicity distributions of the GCs and the HGCs, respectively. In the Left panel, the sample includes all the GCs which have spectroscopic metallicity from the literature (Huchra et al., 1991; Barmby et al., 2000; Perrett et al., 2002; Galleti et al., 2009; Caldwell et al., 2011) and Paper I as well as this work. 

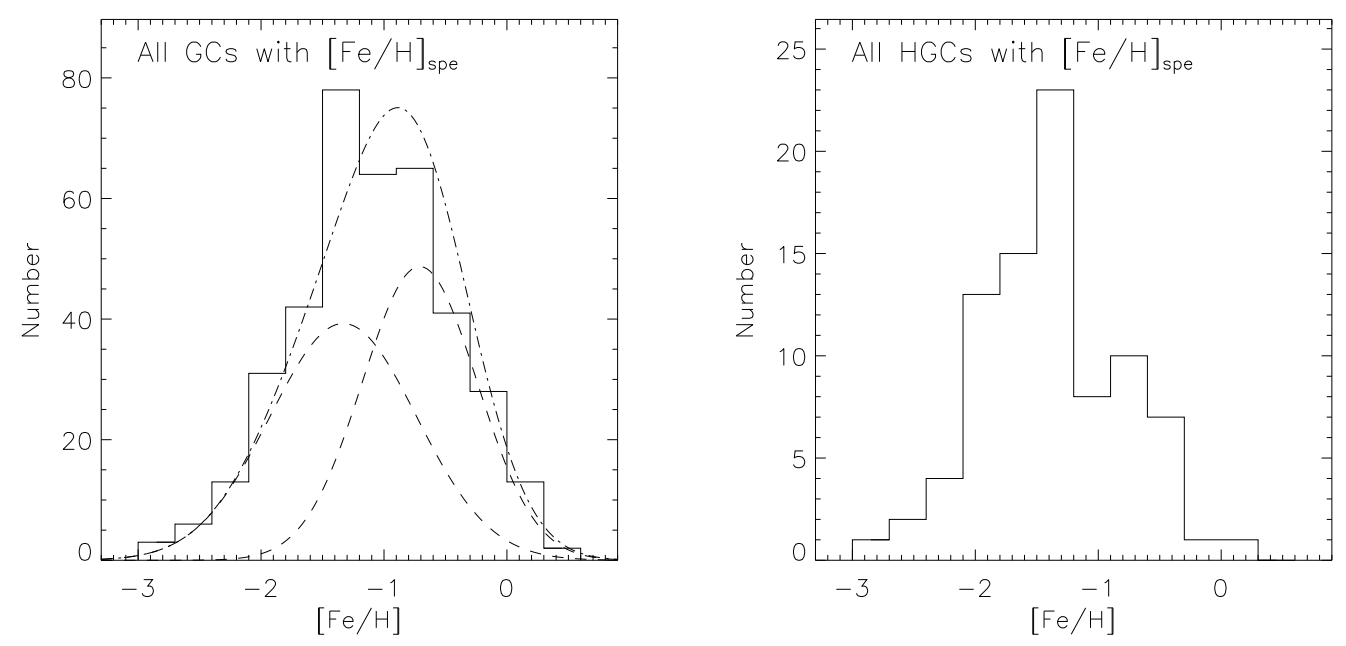

Fig. 8 Metallicity distributions with bin size of 0.3 dex. Left: all the GCs with spectroscopic metallicities. The mixture-model KMM test was applied to divide them to two groups. Right: all the HGCs with spectroscopic metallicities.

In total, there are $386 \mathrm{GCs}$ with spectroscopic metallicity in the distribution. We applied the mixturemodel KMM algorithm to the dataset and it returns an insignificant bimodality with $p$-value $=0.369$, which means that a bimodal distribution is preferred over a unimodal one at $63.1 \%$ confidence level. The numbers of the metal-poor group and the metal-rich group are $N 1=196, N 2=190$, respectively and the mean values of the two groups are $[\mathrm{Fe} / \mathrm{H}]_{1}=-1.43\left(\sigma_{1}^{2}=0.327\right)$ and $[\mathrm{Fe} / \mathrm{H}]_{2}=-0.73$ $\left(\sigma_{2}^{2}=0.215\right)$, respectively. As we can see from the plot, the proportion of the metal-poor and metal-rich group is $\sim 1: 1$, which is lower than the published results. The reason why the bimodal case becomes more insignificant with larger sample may be that more intermediate metallicity GCs, which is between the two metallicity peaks, have been discovered and those intermediate metallicity GCs cause the distribution to be unlikely a bimodal or trimodal distribution. Therefore, the previous works found that the metallicity distributions of M31 GCs is like that of the Milky Way and more recent works with more data show that they are less similar to each other, which may indicate that the formations of the two GC system was substantially different. In the Right panel, it show the metallicity distribution of the HGCs and obviously the metal-poor GCs dominate in the distribution.

As the M31 GCs have been divided into two different groups by the KMM test in the metallicity distribution of Figure 8, we would like to examine the spatial distributions of the two groups with different metallicity. Figure 9 plots the spatial distributions of the metal-rich and metal-poor groups. Note that the metal-poor group appear to occupy a more extended halo and much more widely spatially distributed while the metal-rich group is more centrally concentrated, which is consistent with the conclusions of Perrett et al. (2002); Fan et al. (2008).

Since we have the age estimates of the halo GCs in M31, we are curious about whether the spatial distributions of the young and old populations are the same or not. Here we used the definition of "old population" for age $>2 \mathrm{Gyr}$ and the "young population" for age $<2 \mathrm{Gyr}$ as that did in Caldwell et al. (2009). For the purpose of enlarging our sample, the age estimates for M31 GCs in Fan et al. (2010) and Paper I are also merged into our sample. Figure 10 plots the young and old population spatial distributions, respectively. It is obvious that the young population is more centrally concentrated and it traces the disk shape of the galaxy well. However, the spatial distribution of the old population is more dispersive and it seems that they do not trace the disk shape of the galaxy. 

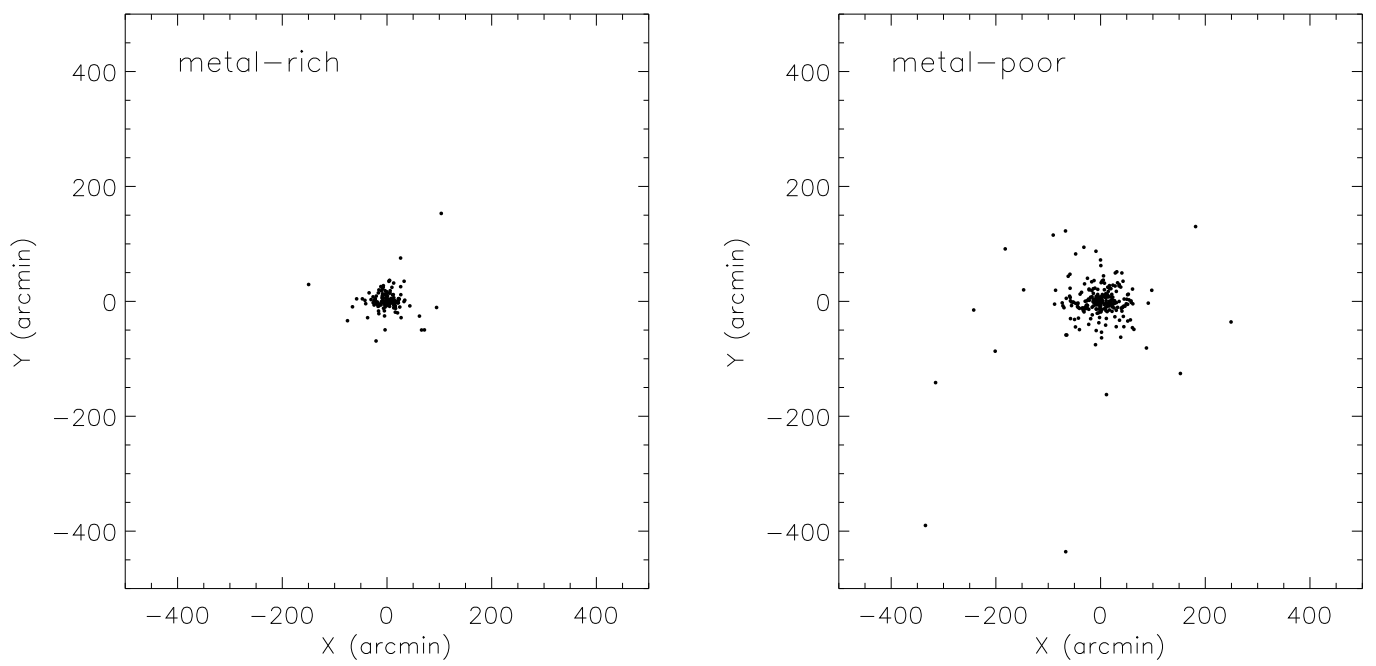

Fig. 9 The spatial distributions of HGCs with different metallicities. Left: metal-rich GCs; Right: metal-poor GCs. The two groups were divided by the KMM test of Figure 8 .
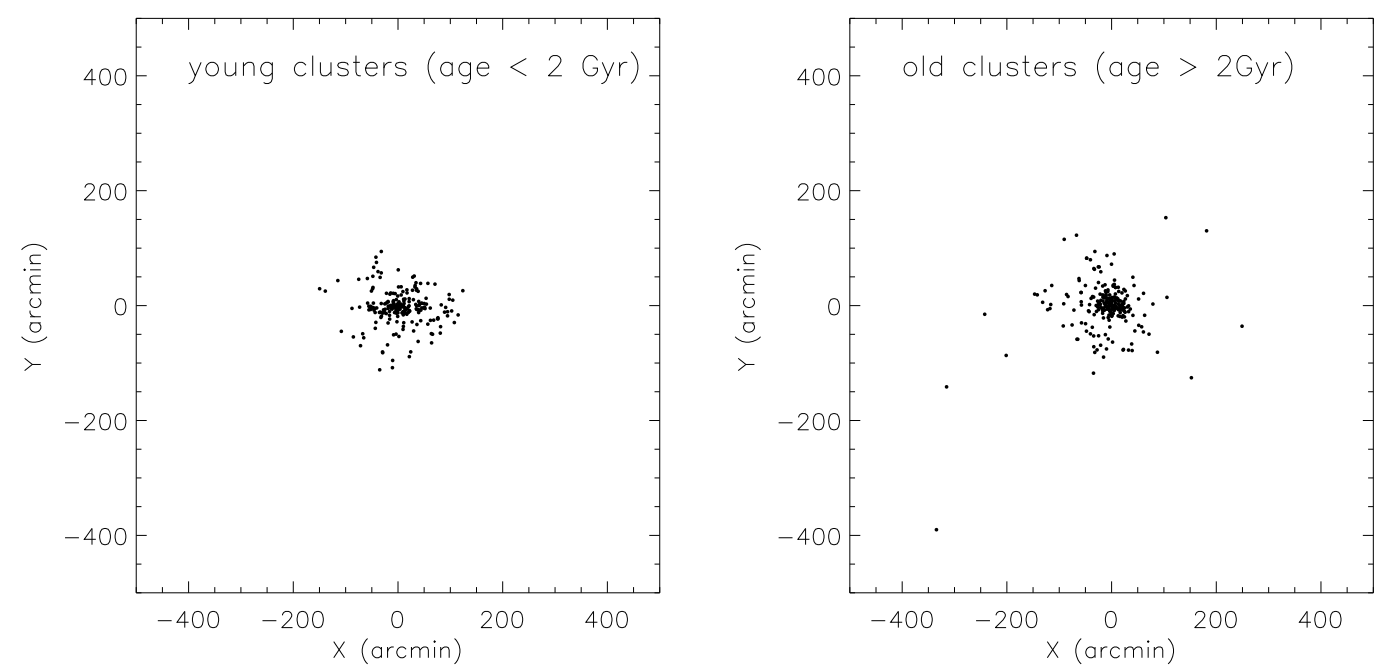

Fig. 10 The spatial distributions of HGCs with young and old populations, respectively. Left: young clusters with age $<2 \mathrm{Gyr}$; Right: old clusters with age $>2$ Gyr.

\section{SUMMARY AND CONCLUSIONS}

This is the second paper of our serial works for M31 halo globular clusters. In Paper I, we mainly focus on the fits method and the metallicity gradient for the M31 GC sample. In this paper, we focus 
on the dynamics, metallicity and age, and their distributions as well as the relationships between these parameters.

We selected eight more confirmed and bright GCs in the halo of M31 from RBC v.4 and observed them with the OMR spectrograph on $2.16 \mathrm{~m}$ telescope at Xinglong site of NAOC in the fall of 2011. These star clusters are located in the halo of galaxy at a projected radius of $\sim 14$ to $\sim 117 \mathrm{kpc}$ from the galactic center, where the sky background is dark so that they can be observed in high signal-to-noise ratio.

For all our sample clusters, we measured all 25 Lick absorption-line indices (see the definitions in, Worthey et al., 1994a; Worthey \& Ottaviani, 1997) and fitted the radial velocities. We found that distributions of the confirmed GCs and the halo GCs are basically symmetric to the systematic velocity of the galalxy.

Similar to Sharina et al. (2006) and our Paper I, we applied the $\chi^{2}$-minimization method to fit the Lick absorption line indices with the updated Thomas et al. (2010) stellar population model in two stellar evolutionary tracks of Cassisi and Padova, separately. The fitting results show that most of our sample clusters are older than 10 Gyr except B290 5.5 Gyr and most of them are metal-poor with metallicity $[\mathrm{Fe} / \mathrm{H}]<-1$ dex except $\mathrm{H} 11$ and H18, suggesting that these halo star clusters were born at the early stage of the galaxy's formation

Again, we would like to study the metallicity gradient of the halo GCs by merging more spectroscopic metallicity from our work, Paper I and the literature. We only considered outer halo clusters with

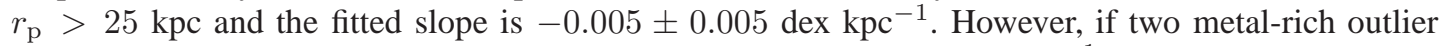
clusters G001 and H11 are excluded, the slope is $-0.002 \pm 0.003 \mathrm{dex} \mathrm{kpc}^{-1}$, which does not change significantly. Furthermore, in order to eliminate the effect the errors of different observations, we added the random errors from $\sigma=-0.5$ to 0.5 to the data and refit the slope agian for ten times. The result shows that the simulated errors do not affact the slope much. Thus it seems that metallicity gradient for M31 outer halo clusters dose not exist, which agrees well with the previous findings (Huxor et al., 2011) and Paper I. This result may imply that the "fragments merging" scenario is dominated in the outer halo of the galaxy beyond $25 \mathrm{kpc}$ from the center during the early stage of the galaxy formation.

We do not find a relationship between metallicity and the radial velocity for M31 GCs sample. It seems that the metallicity is not a function of age for the GCs with age $<7$ Gyr while for the old GCs with age $>7 \mathrm{Gyr}$ there seems to be a trend that the older ones have lower metallicity. This conclusion is similar to that of Fan et al. (2006), who found a possible general trend of the age-metallicity relation with a large scatter. In addition, we plot metallicity distributions with the largest sample of M31 GCs so far and it shows the bimodality is not significant compared to the previous work. This is also found by Caldwell et al. (2011), who used the newly observed spectroscopic data. We also find that the number of the metal-poor and metal-rich groups becomes comparable while the previous works show that the number of metal-poor group is more than that of the metal-rich one. This may be due to many intermedate metallicty metallicity of Caldwell et al. (2011) have been merged into our sample for our statistics. The spatial distributions shows that the metal-rich group is more centrally concentrated while the metal-poor group is occupy a more extended halo and the young population is centrally concentrated while the old populaiton is more extended spatially to the outer halo. This is easy to be understood as the old GCs are usually metal-poor especially for the halo GCs of M31.

Acknowledgements We are indebted to an anonymous referee for his/her thoughtfull comments and insightful suggestions that improved this paper greatly. The authors are also grateful to the kind staff at

the Xinglong $2.16 \mathrm{~m}$ telescope for the support during the observations. This research was supported by National Natural Science Foundation of China through grants Nos. 11003021, 11073027 and 11073032.

\section{References}

Alves-Brito, A., Forbes, D. A., Mendel, J. T., Hau, G. K. T., Murphy, M. T. 2009, MNRAS, 395, L34

Ashman, K. M., Bird, C. M. 1993, AJ, 106, 2281.

Ashman, K. M., Bird, C. M., Zepf, S. E. 1994, AJ, 108, 2348. 
Barmby, P., Huchra, J., Brodie, J., Forbes, D., Schroder, L., Grillmair, C. 2000, AJ, 119, 727

Barmby, P., \& Huchra, J. P. 2001, AJ, 122, 2458

Bono, G., Caputo, F., Cassisi, S., Castellani, V., Marconi, M. 1997, ApJ, 489, 822

Caldwell, N., Harding, P., Morrison, H., Rose, J. A., Schiavon, R., \& Kriessler, J. 2009, AJ, 137, 94

Caldwell, N., Schiavon, R., Morrison, H., Rose, J., Harding, P. 2011, AJ, 141, 61

Cardiel, N., Gorgas, J., Cenarro, J. Gonzalez, J. J. 1998, A\&AS, 127, 597

Cassisi S., Castellani M., Castellani, V. 1997, A\&A, 317, 10

Eggen, O. J., Lynden-Bell, D., Sandage, A. R. 1962, ApJ, 136, 748

Fan, Z., Ma, J., de Grijs, R., Yang, Y., Zhou, X. 2006, MNRAS, 371, 1648

Fan, Z., Ma, J., de Grijs, R., Zhou, X. 2008, MNRAS, 385, 1973

Fan, Z., de Grijs, R., Zhou, X. 2010, ApJ, 725, 200

Fan, Z., et al. 2011, RAA, 11, 1298 (Paper I)

Galleti, S., Federici, L., Bellazzini, M., Fusi Pecci, F., Macrina, S. 2004, A\&A, 426, 917

Galleti, S., Federici, L., Bellazzini, M., Buzzoni, A., Fusi Pecci, F. 2006, A\&A, 456, 985

Galleti, S., Bellazzini, M., Federici, L., Buzzoni, A., Fusi Pecci, F. 2007, A\&A, 471, 127

Galleti, S., Bellazzini, M., Buzzoni, L., Federici, L., Fusi Pecci, F. 2009, A\&A, 508, 1285

Huchra, J. P., Stauffer, J., van Speybroeck, L. 1982, ApJ, 259, L57

Huchra J. P., Brodie J. P., Kent S. M. 1991, ApJ, 370, 495

Huxor, A., Tanvir, N. R., Irwin, M. J., Ferguson, A. M. N., Ibata, R. A., Lewis, G. F., Bridges, T. 2004, in ASP

Conf. Ser. 327, Satellites and Tidal Streams, ed. F. Prada, D. Martinez-Delgado, \& T. Mahoney (San Francisco: ASP), 118

Huxor, A. P., Tanvir, N. R., Irwin, M. J., Ibata, R., Collett, J. L., Ferguson, A. M. N., Bridges, T., Lewis, G. F. 2005, MNRAS, 360, 1007

Huxor, A. 2007, Ph.D. Thesis, Univ. of Hertfordshire, UK

Huxor, A. P., Ferguson, A. M. N., Tanvir, N. R., Irwin, M. J., Mackey, A. D., Ibata, R. A., Bridges, T., Chapman, S. C., Lewis, G. F. 2011, MNRAS, 414, 770

Ma, J., Wu, Z., Wang, S., Fan, Z., Zhou, X., Wu, J., Jiang, Z., Chen, J. 2010, PASP, 122, 1164

Mackey, A. D., et al. 2006, ApJ, 653, L105

Mackey, A. D., et al. 2007, ApJ, 655, L85

Mackey, A. D., et al. 2010, MNRAS, 401, 533

Maraston, C. 1998, MNRAS, 300, 872

Massey, P., Strobel, K., Barnes, J. V., Anderson, E. 1988, ApJ, 328, 315

McConnachie, A. W., Irwin, M. J., Ferguson, A. M. N., Ibata, R. A., Lewis, G. F., Tanvir, N. 2005, MNRAS, 356, 979

Peng, E., et al. 2006, ApJ, 639, 838

Perina, S., et al. 2010, A\&A, 511, 23

Perrett, K. M., Bridges, T. J., Hanes, D. A., Irwin, M. J., Brodie, J. P., Carter, D., Huchra, J. P., Watson, F. G. 2002, AJ,123, 2490

Racine, R. 1991, AJ, 101, 865

Salpeter, E. E. 1955, ApJ, 121, 161

Searle L., Zinn R. 1978, ApJ, 225, 357

Sharina, M. E., Afanasiev, V. L., Puzia, T. H. 2006, MNRAS, 372, 1259

Thomas, D., Maraston, C., Bender, R. 2003, MNRAS, 339, 897

Thomas, D., Maraston, C., Johansson, J. 2010, arXiv1010.4569.

Thomas, D., Maraston, C., Korn, A. 2004, MNRAS, 351, L19

van den Bergh, S. 1969, ApJS, 19, 145

Worthey, Guy, Faber, S. M., Gonzalez, J. J., Burstein, D. 1994, ApJS, 94, 687

Worthey, G. 1994, ApJS, 95, 107

Worthey, Guy, Ottaviani, D. L. 1997, ApJS, 111, 377 